\title{
Rainbow Ramsey theorems for colorings establishing negative partition relations
}

by

András Hajnal (Budapest)

\begin{abstract}
Given a function $f$, a subset of its domain is a rainbow subset for $f$ if $f$ is one-to-one on it. We start with an old Erdős problem: Assume $f$ is a coloring of the pairs of $\omega_{1}$ with three colors such that every subset $A$ of $\omega_{1}$ of size $\omega_{1}$ contains a pair of each color. Does there exist a rainbow triangle? We investigate rainbow problems and results of this style for colorings of pairs establishing negative "square bracket" relations.
\end{abstract}

1. Introduction and history. Anti-Ramsey theorems appeared probably for the first time in the 1973 paper [9] of Richard Rado, claiming the existence of subsets with elements of different colors of the domain of a given coloring. Later in the game, the more expressive name of rainbow subset was coined. In this paper we will mostly consider 2-partitions, i.e. colorings $f$ of unordered pairs of a set. A subset of pairs will be called a rainbow subset (for $f$ ) if $f$ is one-to-one on it. Our starting point will be a problem of Paul Erdős, stated long before any of these names were coined:

Assume $f:\left[\omega_{1}\right]^{2} \rightarrow 3$ is a 2-partition of $\omega_{1}$ with three colors such that each subset $A \subseteq \omega_{1}$ of size $\omega_{1}$ contains a pair of each color. Does there exist a rainbow triangle for $f$ ?

This is Problem 68 of [3] written in 1967. We restate it in the jargon of partition relations developed in [5]:

Problem 1.1. Assume $f:\left[\omega_{1}\right]^{2} \rightarrow 3$ establishes $\omega_{1} \nrightarrow\left[\omega_{1}\right]_{3}^{2}$. Does there exist a rainbow triangle for $f$ ?

We knew that the answer is affirmative under some stronger conditions e.g.

2000 Mathematics Subject Classification: Primary 03E05.

Key words and phrases: partition relation, rainbow subset, coloring.

Research partially supported by Hungarian National Research Grants T 61600 and K 68262 . 
FACT 1.2. Assume $f:\left[\omega_{1}\right]^{2} \rightarrow 3$ establishes $\omega_{1} \nrightarrow \rightarrow\left[\left(\omega, \omega_{1}\right)\right]_{3}^{2}$ (i.e. for $A \in\left[\omega_{1}\right]^{\omega}$ and $B \in\left[\omega_{1}\right]^{\omega_{1}}$, $f$ takes all three values on $\left.[A, B]^{1,1}\right)$. Then there exists a rainbow triangle for $f$.

However, in those early days, we could only construct an $f$ satisfying the condition of 1.2 using $\mathrm{CH}$.

Definition 1.3. For a coloring $d:[k]^{2} \rightarrow \omega_{1}, k \leq \omega$ we write $d \Rightarrow f$ if there is a one-to-one map $\Phi: k \rightarrow \omega_{1}$ such that

$$
d(\{n, m\})=f(\{\Phi(n), \Phi(m)\}) \quad \text { for } n, m \in k .
$$

We could generalize 1.2 to

FACT 1.4. Assume $f:\left[\omega_{1}\right]^{2} \rightarrow \omega_{1}$ establishes $\omega_{1} \nrightarrow\left[\left(\omega, \omega_{1}\right)\right]_{\omega_{1}}^{2}$. Then $d \Rightarrow f$ for an arbitrary $d:[\omega]^{2} \rightarrow \omega_{1}$.

As already mentioned, we were not able to verify in ZFC that this does not hold vacuously and it bothered us that we could not lift it e.g. replacing $\omega, \omega_{1}$ by $\omega_{1}, \omega_{2}$ respectively. The next steps were taken in a paper of Shelah [10] written in 1975. He proved

THEOREM 1.5 (Shelah [10]).

1. CH implies that 1.1 fails for some $f$ with $\omega$ colors.

2. $\diamond$ implies that 1.1 fails for an $f$ with $\omega_{1}$ colors.

Shelah also showed in [10] that a possible "lifting" of Fact 1.4 is consistently false say adding one Cohen real to a model of GCH. In more detail, he constructed a graph of size $\omega_{1}$ from the Cohen real which does not embed isomorphically into any graph of the ground model. Then any graph of the ground model establishing the partition relation $\omega_{2} \nrightarrow\left[\left(\omega_{2}, \omega_{1}\right)\right]_{\omega_{1}}^{2}$ satisfies the same relation in the new model, and we have a graph of size $\omega_{1}$ that does not embed into it.

Knowing all this, in our 1978 paper [2] we stated implicitly a generalization of 1.4.

Theorem $1.6([2])$. Assume that $f$ establishes $\omega_{1} \not \rightarrow\left[\left(\omega_{1} ; \omega_{1}\right)\right]_{\omega}^{2}$. Then $d \Rightarrow f$ for an arbitrary $d:[\omega]^{2} \rightarrow \omega$.

The symbol with the semi-colon ";" means that all $\omega_{1}$ by $\omega_{1}$ "half-graphs" are totally multicolored, i.e. for all $A, B \subseteq \omega_{1}$ with $|A|=|B|=\omega_{1}$ and $n<\omega$ there are $\alpha \in A$ and $\beta \in B$ with $\alpha<\beta$ such that $f(\{\alpha, \beta\})=n$. I want to mention that [2] seems to be the first paper in print where this important concept was used. I think it was invented (discovered) by Fred Galvin. The following was proved 37 years later by Justin Moore:

TheOrem 1.7 (Moore [7]). (ZFC) There is an $f$ establishing

$$
\omega_{1} \nrightarrow\left[\left(\omega_{1} ; \omega_{1}\right)\right]_{\omega_{1}}^{2} \text {. }
$$


This is a byproduct of Moore's result [7] showing the existence of $L$ spaces in ZFC. All the above justifies revisiting the old Problem 1.1.

2. $\nRightarrow$ relations. First we remark that we still do not know if the conclusions of either clauses of Theorem 1.5 can be proved under weaker conditions. Next we want to show that a Theorem 1.7 type generalization cannot hold if we only assume that each $[A]^{2}$ with $|A|=\omega_{1}$ is totally multicolored.

Theorem 2.1. There exist a rainbow $d:[4]^{2} \rightarrow 6$ and an $f:\left[\omega_{1}\right]^{2} \rightarrow 6$ establishing $\omega_{1} \nrightarrow\left[\omega_{1}\right]_{6}^{2}$ such that

$$
d \nRightarrow f \text {. }
$$

Proof. First we define $e:[4]^{2} \rightarrow W$ and $g:\left[\omega_{1}\right]^{2} \rightarrow W$ where

$$
W=\{(+,+),(+,-),(-,+),(-,-)\} .
$$

Let

$$
\begin{array}{lll}
e(\{0,1\})=(+,-), & e(\{1,2\})=(-,+), & e(\{2,3\})=(+,-), \\
e(\{0,3\})=(-,+), & e(\{0,2\})=(+,+), & e(\{1,3\})=(-,-) .
\end{array}
$$

Let $<_{R}$ and $<_{A}$ be real and Aronszajn type orderings of $\omega_{1}$. For $\alpha<$ $\beta<\omega_{1}$ let $g(\alpha, \beta)=(u, v)$ with $u, v \in\{+,-\}$, where $u=+$ iff $\alpha<_{A} \beta$, and $v=+$ iff $\alpha<_{R} \beta$.

It is a well known property of these orderings that for all $B \in\left[\omega_{1}\right]^{\omega_{1}}$ there are $C, D, E, F \in[B]^{\omega_{1}}$ such that $C<_{A} D, C<_{R} D, E<_{A} F$ and $F<_{R} E$. This implies that each $B \in\left[\omega_{1}\right]^{\omega_{1}}$ contains a complete $\omega_{1}$ by $\omega_{1}$ half-graph for $g$ in each of the colors in $W$.

It is an easy exercise to see that $e \nRightarrow g$. Let now $h$ be as in Moore's Theorem 1.7. Then $k=(g, h)$ establishes $\omega_{1} \nrightarrow\left[\omega_{1}\right]_{\omega_{1}}^{2}$. Using $k$ and $e$ it is a matter of easy calculation to get $f$ and $d$ as required in the theorem.

Next we are going to investigate the cases when $f$ establishes

$$
\omega_{1} \nrightarrow\left[\left(\omega_{1}, \omega_{1}\right)\right]_{\gamma}^{2}
$$

i.e. all $\omega_{1}$ by $\omega_{1}$ subgraphs are totally multicolored for some $\gamma$.

FACT 2.2. Assume $f$ establishes $\omega_{1} \nrightarrow\left[\left(\omega_{1}, \omega_{1}\right)\right]_{3}^{2}$. Let $d:[3]^{2} \rightarrow 3$ be one-to-one. Then $d \Rightarrow f$, i.e. all possible rainbow triangles exist.

Proof. The assumption implies that for some $\alpha \in \omega_{1}$ both sets

$$
\left\{\beta \in \omega_{1}: f(\alpha, \beta)=d(0,1)\right\}, \quad\left\{\gamma \in \omega_{1}: f(\alpha, \gamma)=d(0,2)\right\}
$$

are of cardinality $\omega_{1}$.

FACT 2.3. There exist a rainbow $d:[5]^{2} \rightarrow 10$ and an $f:\left[\omega_{1}\right]^{2} \rightarrow 10$ establishing $\omega_{1} \nrightarrow\left[\left(\omega_{1}, \omega_{1}\right)\right]_{10}^{2}$ such that

$$
d \nRightarrow f \text {. }
$$


Proof (outline). Define $e:[5]^{2} \rightarrow 2$ by the stipulation

$$
e(\{i, j\})=0 \quad \text { for } i<5 \text { and } j \equiv i+1 \bmod 5 .
$$

That is, $e$ is a "pentagon without a diagonal". Let $d:[5]^{2} \rightarrow 10$ be oneto-one such that $d(\{i, i+1\})<5$ iff $e(\{i,, i+1\})=0$. Let $<_{R}$ be a real type ordering of $\omega_{1}$. Let $g(\alpha, \beta):\left[\omega_{1}\right]^{2} \rightarrow 2$ be the "Sierpiński" partition, that is, $g(\alpha, \beta)=0$ iff $\alpha<_{R} \beta$ for $\alpha<\beta<\omega_{1}$. It is well known that every complete bipartite $\omega_{1}$ by $\omega_{1}$ half-graph contains a complete bipartite $\omega_{1}$ by $\omega_{1}$ half-graph in both colors for $g$. Again by Moore's theorem, we can take an $h$ establishing $\omega_{1} \nrightarrow\left[\left[\left(\omega_{1} ; \omega_{1}\right)\right]_{5}^{2}\right.$. Set $f=g \cdot 5+h$. Then $f$ establishes $\omega_{1} \nrightarrow\left[\left(\omega_{1}, \omega_{1}\right)\right]_{10}^{2}$ and $d \Rightarrow f$ would imply $e \Rightarrow g$, which is known to be false.

Problem 2.4. Can we improve 2.3 to have $a d:[4]^{2} \rightarrow 6$ and an $f$ establishing $\omega_{1} \nrightarrow\left[\left(\omega_{1}, \omega_{1}\right)\right]_{6}^{2}$ ?

\section{Rainbow theorems}

Theorem 3.1. Assume $f:\left[\omega_{1}\right]^{2} \rightarrow \omega$ establishes $\omega_{1} \nrightarrow\left[\left(\omega_{1}, \omega_{1}\right)\right]_{\omega}^{2}$. Then there exists an infinite rainbow set.

Proof. We use $A, B, C, \ldots$ to denote subsets of $\omega_{1}$ of size $\omega_{1}$, and $N, M, \ldots$ to denote infinite subsets of $\omega$; moreover, we set

$$
f_{j}(x)=\left\{y \in \omega_{1}: f(x, y)=f(\{x, y\})=j\right\}
$$

for $j<\omega$ and $x \in \omega_{1}$.

3.1.1. Assume $B \cap C=\emptyset$ and

$$
\forall n \in M \forall x \in B\left(\left|f_{n}(x) \cap C\right| \leq \omega\right) .
$$

Then

$$
\forall n \in M \forall C^{\prime} \subseteq C \exists y \in C^{\prime}\left(\left|f_{n}(y) \cap B\right|=\omega_{1}\right) .
$$

Otherwise we could pick, by transfinite induction, a pair $\left(B^{\prime}, C^{\prime \prime}\right)$ omitting the color $n$.

Let $(*)(A, N)$ be the following property of $A$ and $N$ : There are $B, C \subseteq A$ and $M \subseteq N$ such that

$$
\forall B^{\prime} \subseteq B \forall C^{\prime} \subseteq C \forall m \in M \exists x \in B^{\prime}\left(\left|f_{m}(x) \cap C^{\prime}\right|=\omega_{1}\right) .
$$

When $(*)(A, N)$ holds we denote by

$$
B(A, N), C(A, N), M(A, N)
$$

the relevant sets $B, C, M$ respectively, with $B \cap C=\emptyset$.

3.1.2. Assume that for some $A_{0}, N_{0},(*)(A, N)$ holds for all $A \subseteq A_{0}$ and $N \subseteq N_{0}$. Then there is an infinite rainbow subset. 
Define $A_{k}, B_{k}, N_{k}$ by induction on $k<\omega$. Assume $A_{k}, N_{k}$ are defined. Let $B_{k}=B\left(A_{k}, N_{k}\right), A_{k+1}=C\left(A_{k}, N_{k}\right), N_{k+1}=N\left(A_{k}, N_{k}\right)$. Let $\left\{N_{k}^{\prime}: k<\omega\right\}$ be a disjoint refinement of $\left\{N_{k}: k<\omega\right\}$ and let

$$
N_{k}^{\prime}=\left\{n_{i}^{k}: i<\omega\right\}
$$

be a one-to-one enumeration of $N_{k}^{\prime}$ for $k<\omega$. It is now easy to pick $x_{i} \in A_{i}$ for $i<\omega$ in such a way that $c\left(x_{i}, x_{j}\right)=n_{j}^{i}$ for $i<j<\omega$. This proves 3.1.2, as $\left\{x_{i}: i<\omega\right\}$ is an infinite rainbow set.

Hence to finish the proof of Theorem 3.1 it is sufficient to prove

3.1.3. Assume $(*)(A, N)$ is false for some $A$ and $N$. Then $A$ has an infinite rainbow subset.

Let $N=\bigcup_{k<\omega} N_{k}, A=\bigcup_{k<\omega} A_{k}$ be disjoint partitions. To prove 3.1.3 we first prove

3.1.4. There are $x \in A_{0}$ and $\left\{n_{i} \in N_{0}: 1 \leq i<\omega\right\}$ one-to-one such that

$$
\left|f_{n_{i}}(x) \cap A_{i}\right|=\omega_{1} \quad \text { for } 1 \leq i<\omega .
$$

For an $x \in A_{0}$ we try to choose $n_{i}, 1 \leq i<\omega$, by induction on $i$. Assume we have chosen $n_{k}, 1 \leq k \leq i$, with $\left|f_{n_{k}}(x) \cap A_{k}\right|=\omega_{1}$. If there is always an $n$ such that

$$
n \in N_{0} \backslash\left\{n_{k}: 1 \leq k \leq i\right\} \quad \text { and } \quad\left|f_{n}(x) \cap A_{i+1}\right|=\omega_{1}
$$

we can choose $n_{i+1}$ to be the smallest of these and 3.1.3 is true. If not, let $i(x)$ be the smallest $i$ for which $(+)$ fails. If $(+)$ fails for all $x \in A_{0}$ then for some $1 \leq i<\omega$ and $M=N_{0} \backslash\left\{n_{k}: 1 \leq i\right\}$,

$$
C=\left\{x \in A_{0}: i(x)=i\right\}
$$

has cardinality $\omega_{1}$. Choosing $B=A_{i+1}$ we find that

$$
\left|f_{n}(x) \cap B\right| \leq \omega \quad \text { for } n \in M \text { and } x \in C .
$$

But then, by 3.1.2, for all $n \in M$ there is $x \in B$ with $\left|f_{n}(x) \cap C\right|=\omega_{1}$, a contradiction to the assumption that $(*)(A, N)$ is false. This shows 3.1.4. To finish the proof of 3.1.3 and Theorem 3.1, we can use 3.1.4 inductively.

Here is a problem that has not been looked at very thoroughly:

Problem 3.2. Under the conditions of 3.1 , is there a rainbow set containing all the colors?

Theorem 3.3. For every $1<k<\omega$ there is an $n \in \omega$ with $\left(\begin{array}{c}k \\ 2\end{array}\right) \leq n$ such that every $f$ satisfying $\omega_{1} \nrightarrow\left[\left(\omega_{1}, \omega_{1}\right)\right]_{n}^{2}$ has a rainbow set of size $k$.

Proof. We prove the following statement by induction on $2 \leq k<\omega$. There is an $n<\omega$ such that if $\operatorname{Dom}(f) \subseteq\left[\omega_{1}\right]^{2}$ satisfies $\omega_{1} \nrightarrow\left[\left(\omega_{1}, \omega_{1}\right)\right]_{n}^{2}$ (note that this means that for all $A, B \subseteq \omega_{1}$ with $|A|=|B|=\omega_{1}$ and for all $i<n$ there are $\alpha \in A$ and $\beta \in B$ with $\{\alpha, \beta\} \in \operatorname{Dom}(f)$ such that 
$f(\{\alpha, \beta\})=i)$ and $\left\{A_{i}: i<n\right\}$ are pairwise disjoint subsets of $\omega_{1}$ of size $\omega_{1}$, then there is a rainbow partial transversal $P\left([P]^{2} \subseteq \operatorname{Dom}(f)\right)$ of size $k$ for these sets. Just as in the proof of 3.1, put

$$
f_{j}(x)=\left\{y \in \omega_{1}: f(x, y)=f(\{x, y\})=j\right\}
$$

for $j<\omega$ and $x \in \omega_{1}$. Assume $n$ is good for $k$ and $A_{0}, \ldots, A_{2 n-1}$ are pairwise disjoint subsets of $\omega_{1}$ of size $\omega_{1}$ with union $A$.

Let $(*)$ denote the following statement: There are $x, i_{x}, N_{x}, \varphi_{x}$ such that $x \in A_{i_{x}}, N_{x} \subseteq 2 n \backslash\left\{i_{x}\right\}, \varphi_{x}: N_{x} \rightarrow 2 n$ is one-to-one,

$$
\left|f_{\varphi_{x}(j)}(x) \cap A_{j}\right|=\omega_{1} \quad \text { for } j \in N_{x},
$$

and $\left|N_{x}\right|=n$. If $(*)$ holds for an $x$ then applying the induction hypothesis for the sets

$$
f_{\varphi_{x}(j)}(x) \cap A_{j}, \quad j \in N_{x},
$$

and for the color set $2 n \backslash \varphi\left[N_{x}\right]$ we get a rainbow partial transversal of size $k$ for these sets, and adding $x$ to it we get a rainbow transversal of size $k+1$ for the sets $A_{0}, \ldots, A_{2 n-1}$.

If $(*)$ is false, choosing an $N_{x}$ of maximal size for $x \in A$ we will have $\left|N_{x}\right| \leq n-1$ for $x \in A$. By thinning out, we get sets $B_{i} \subseteq A_{i}, i<2 n$, of size $\omega_{1}$ and $N_{i}, M_{i} \subseteq 2 n, i<2 n$, such that $N_{x}=N_{i}$ and $M_{i}=\varphi_{x}\left[N_{i}\right]$ for $x \in B_{i}$ for $i<2 n$.

Then $i \mapsto N_{i}$ is a set mapping of order at most $n-1$ on $2 n$. By a theorem of de Bruijn and Erdős, from 1951, there are $i \neq j$ such that $i \notin N_{j}$ and $j \notin N_{i}$. As $\left|M_{i} \cup M_{j}\right|<2 n$ we can choose $l \notin M_{i} \cup M_{j}$. By the maximality of $N_{i}$ we know that $\left|f_{l}(x) \cap B_{j}\right| \leq \omega$ for $x \in B_{i}$ and likewise $\left|f_{l}(x) \cap B_{i}\right| \leq \omega$ for $x \in B_{j}$. We could then pick, by an easy transfinite induction, sets $C_{i} \subseteq B_{i}$ and $C_{j} \subseteq B_{j}$, both of size $\omega_{1}$, such that the color $l$ is missing from the bipartite $\left(\omega_{1}, \omega_{1}\right)$ determined by $C_{i}$ and $C j$. This contradicts the assumption.

Corollary 3.4. In Theorem $3.3, n$ can be chosen to be $2^{k-2}$ for $2 \leq$ $k<\omega$.

Problem 3.5. Can $n$ be taken to be $\left(\begin{array}{l}k \\ 2\end{array}\right)$ in Theorem 3.3 ?

4. Resurrecting the problem for larger cardinals. We explained in Section 1 how Shelah's example described in 1.5 forced us to consider problems only for underlying sets of size at most $\omega_{1}$. In [2] written in 1978 we tried to ask if we can get every graph of size $\omega_{1}$ as an induced subgraph provided the graph shows $\omega_{2} \nrightarrow\left[\left(\omega_{1}, \omega\right)\right]_{\omega_{1}}^{2}$, a stronger assumption that one can only make consistent. Recently Soukup showed that the simple method of adding one Cohen real gives a negative answer as well. Working through the material of this paper I realized that this trick only kills questions of $\Rightarrow$ type. The following is probably the simplest problem I cannot solve: 
Problem 4.1. Assume $G C H$ and let $f$ establish

$$
\omega_{2} \nrightarrow\left[\left(\omega_{1}, \omega_{2}\right)\right]_{\omega_{1}}^{2} .
$$

Does there exist a rainbow subset of size $\omega_{1}$ for $f$ ?

In fact, we do not know a single case where for some $\kappa>\lambda>\omega$ some $f$ : $[\kappa]^{2} \rightarrow \lambda$ establishes $\kappa \not\left[[(\kappa, \kappa)]_{\lambda}^{2}\right.$ and for all such $f$ there is an uncountable rainbow set.

5. Finitary problems. In our paper [4] we considered finitary Ramsey problems and proved in 1989

Theorem 5.1 (Erdős-Hajnal [4, Theorem 1.3]). Assume $2 \leq k, s<\omega$ and $d:[k]^{2} \rightarrow s$. Then there are $n_{0}$ and a real number $r>0$ such that for all $f:[n]^{2} \rightarrow s$ establishing

$$
n \nrightarrow\left[e^{r \sqrt{\log n}}\right]_{s}^{2}
$$

$d \Rightarrow f$ holds.

In fact, we only wrote down the proof of this result for $s=2$. Janos Pach kindly communicated to us that he can prove a much stronger result for a great many cases. Most relevant to this paper, he can prove:

THEOREM 5.2 (Fox-Pach [6]). There are $n_{0}$ and $\varepsilon>0$ such that for any $n>n_{0}$ and $f$ establishing

$$
n \nrightarrow\left[n^{\varepsilon}\right]_{3}^{2}
$$

there is a rainbow triangle for $f$.

\section{References}

[1] P. Erdős, F. Galvin and A. Hajnal, On set-systems having large chromatic number and not containing prescribed subsystems, in: Colloq. Math. Soc. J. Bolyai 10, NorthHolland, 1975, 425-513.

[2] P. Erdős and A. Hajnal, Embedding theorems for graphs establishing negative partition relations, Period. Math. Hungar. 9 (1978), 205-230.

[3] —, 一, Unsolved problems in set theory, in: Proc. Sympos. Pure Math. 13, Part I, Amer. Math. Soc., Providence, RI, 1971, 17-48.

[4] —, - Ramsey type theorems, Discrete Appl. Math. 25 (1989), 39-52.

[5] P. Erdős, A. Hajnal, A. Máté and R. Rado, Combinatorial Set Theory: Partition Relations for Cardinals, Studies Logic Found. Math. 106, Akadémiai Kiadó and North-Holland, Budapest and Amsterdam, 1984.

[6] J. Fox and J. Pach, Erdös-Hajnal-type results on intersection patterns of geometric objects, Israel J. Math., to appear.

[7] J. T. Moore, A solution to the $L$ space problem and related ZFC constructions, preprint, 2005.

[8] - - A solution to the L space problem, J. Amer. Math. Soc. 9 (2006), 717-736. 
[9] R. Rado, Anti-Ramsey theorems, in: Colloq. Math. Soc. J. Bolyai 10, Vol. III, NorthHolland, 1975, 1159-1168.

[10] S. Shelah, Colouring without triangles and partition relations, Israel J. Math. 20 (1975), 1-12.

Rényi Institute

Reáltanoda u. 13-15

1053 Budapest, Hungary

E-mail: ahajnal@renyi.hu

Received 12 December 2006;

in revised form 13 November 2007 